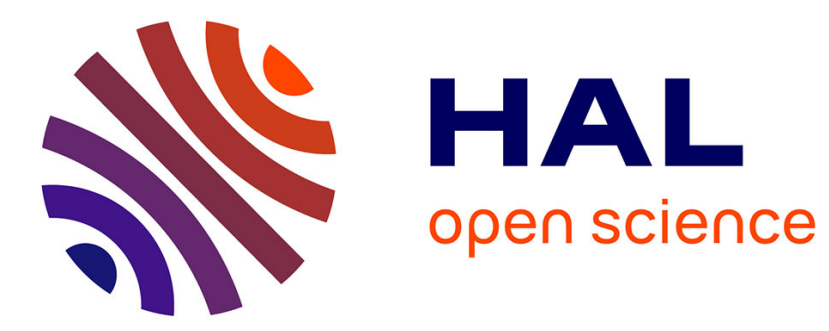

\title{
Digital volume correlation applied to X-ray tomography images from spherical indentation tests on lightweight gypsum
}

\author{
Amine Bouterf, Stéphane Roux, François Hild, Jérôme Adrien, Eric Maire, \\ Sylvain Meille
}

\section{To cite this version:}

Amine Bouterf, Stéphane Roux, François Hild, Jérôme Adrien, Eric Maire, et al.. Digital volume correlation applied to X-ray tomography images from spherical indentation tests on lightweight gypsum. Strain, 2014, 50 (5), pp.444-453. 10.1111/str.12101 . hal-01068402

\section{HAL Id: hal-01068402 https://hal.science/hal-01068402}

Submitted on 25 Sep 2014

HAL is a multi-disciplinary open access archive for the deposit and dissemination of scientific research documents, whether they are published or not. The documents may come from teaching and research institutions in France or abroad, or from public or private research centers.
L'archive ouverte pluridisciplinaire HAL, est destinée au dépôt et à la diffusion de documents scientifiques de niveau recherche, publiés ou non, émanant des établissements d'enseignement et de recherche français ou étrangers, des laboratoires publics ou privés. 


\title{
Digital volume correlation applied to X-ray tomography images from spherical indentation tests on lightweight gypsum
}

\author{
A. Bouterf ${ }^{1,3}$, S. Roux ${ }^{1}$, F. Hild ${ }^{1}$, J. Adrien ${ }^{2}$, E. Maire ${ }^{2}$ and S. Meille ${ }^{2}$ \\ ${ }^{1}$ LMT-Cachan, ENS Cachan/CNRS/UPMC/PRES UniverSud Paris, \\ 61 avenue du Président Wilson, \\ 94235 Cachan Cedex, France \\ 2 MATEIS-INSA de Lyon, Bât. B. Pascal, \\ 7, Avenue Jean Capelle, 69621 Villeurbanne Cedex, France \\ ${ }^{3}$ Saint-Gobain Recherche, 39, quai Lucien Lefranc, \\ B. P. 135, 93303 Aubervilliers Cedex, France
}

\begin{abstract}
Cylinders made of lightweight gypsum are extracted from industrial plasterboard and then indented in-situ in an X-ray tomograph. The results from the in-situ experiment show that a compacted zone develops under the indenter, which is displaying a very sharp boundary with the undamaged material. Tomographic imaging during the mechanical load associated with digital volume correlation enable the displacement fields to be measured during the test. However, because of the inhomogeneous nature of the indentation test, a high spatial resolution for the displacement is called for, and because the range of displacement amplitudes is small, uncertainties on the measured displacement and strain fields are large. In this study, a new methodology is presented to address integrated digital volume correlation based on a library of fields computed from a commercial finite element software. It allows many fluctuations in the estimated displacement fields to be filtered out and the measurement to be much more robust and reliable. This opens new pathways for the identification of mechanical properties.
\end{abstract}

Keywords: Digital volume correlation; global approach; reduced basis; strain field; plaster

\section{Introduction}

Plasterboard is a low-density material, which is composed of lightweight gypsum core lined with paper coating. Two main routes are commonly followed for the production of porous gypsum. In the first method, gas bubbles are formed in the gypsum paste through very rapid mixing and chemical additives. The second one consists of foaming by air entrainment in the wet gypsum paste [1]. The objective of introducing foam in the board slurry is to create voids 
to further reduce the final weight of the board at fixed plaster/water ratio. To improve the adhesion between paper and plaster, starch is added in most cases. This is beneficial to the board flexural strength.

The relationship between the microstructure and the mechanical properties of the core of the board [2,3,4], or the evaluation of the fire resistance of a partition wall made of plasterboard [5] have already been studied in detail. Benouis [6] showed the orthotropic nature of plasterboard, which is a consequence of the tensile behavior of the paper. However, lightweight gypsum as such has received only little recognition as a structural part; its mechanical behavior and fracture resistance are scarcely investigated in the scientific literature. Yet, some mechanical properties are required for boards, and hence a good knowledge of the mechanical degradation is a need. In the sequel, it is proposed to use X-ray tomography to investigate mechanical properties of plasterboards.

X-ray tomography is a powerful non destructive technique allowing the material microstructure to be characterized [7]. It has been used by numerous laboratories to investigate the microarchitecture of trabecular bone [8], to characterize the microstructure of cellular materials [9], to qualitatively reveal damage mechanisms in the bulk of particulate composites [10], to visualize propagation of cracks in fatigue [11]. To make progress in the understanding of deformation and damage mechanisms in samples, it is crucial to be able to observe the latter ones at the same time as they are loaded. The development of in-situ experiments monitored by X-ray tomography started with the observation of Guvenilir et al. [12]. Since then, mechanical testing coupled with in-situ tomographic observations has been developed [8, 13, 14]. The actual microstructures may then be used in finite element calculations in which numerical models are produced to represent as precisely as possible the architecture of these materials [15, 9].

Another way to exploit the reconstructed volumes is to use Digital Volume Correlation (DVC) $[8,16,17]$ to measure displacement fields in the bulk of the sample. DVC is the 3D extension of standard digital image correlation [18]. It can either be "local" (or subset-based) or "global" when global constraints such as continuity are prescribed to the measured fields [19]. The latter approach was used to analyze the correlation between local density and strain levels in stone wool [20], stress intensity profiles in a cracked sample [21] or to identify and validate constitutive and numerical models [22].

In the following, indentation tests will be carried out to analyze the behavior of gypsum. They are relatively easy to perform on a specimen of simple shape and small volume. They can be used to characterize the mechanical behavior of materials. The spherical indenter, compared to other geometries, has two major advantages. The first is the ability to distinguish the transition from the elastic behavior to the elastoplastic behavior, a very interesting feature especially for materials with low yield stress. With conical or pyramidal (i.e. sharp) indenters, plasticity begins as soon as contact is established with the medium. Second, it has been shown that with a pyramidal indenter, several sets of material parameters can give rise to very similar load-displacement curves. The discriminating character is somewhat more pronounced for a spherical indenter [23].

Early works on the indentation of foams have suggested that their response is controlled to a large extent by their compressive strength [24, 25]. A review of the existing literature on indentation tests shows that spherical indentation was used to study ceramics (e.g. alumina whose porosity ranges from $2.5 \%$ to $18 \%$ [26]). The results confirm that a porous material is more susceptible to damage than cracking macroscopically in a brittle manner. Several works have been published on the modeling of the indentation behavior of cellular materials [27, 28]. Some of the studies concern brittle dense rocks and much less attention has been paid to the problem of indentation of porous rocks [29, 30].

$\mathrm{X}$-ray tomography has been applied to the indentation of aluminum-based metallic foams [31]. In the latter study X-ray tomography revealed that the deformation was found 
to be almost restricted to a spherically shape compacted zone under the indenter. Smith and Bay [32] used DVC to quantify strains in aluminum foam and to compare them with simulated fields using an isotropic finite element analysis. DVC has also been used to measure strain fields in spherical indentation of a closed-cell and open-cell aluminium foams [33]. Mostavafi et al. [34] used DVC to study the fracture behavior of a short-bar chevron notch crack propagation specimen made of polygranular nuclear graphite (i.e. a quasi-brittle material). However, in the analyzed cases the strain levels were very small, much smaller than the strain measurement uncertainty.

In this study, cylinders made of lightweight gypsum are tested in-situ by using a spherical indenter. As for other materials (see above), the final aim of this test is to analyze the transition from the elastic behavior to the nonlinear response in the compacted zone. The actual state of strain and stress has to be evaluated. Therefore there is a need for measuring very small strain levels in most of the material and detecting high strain gradients in the transition zone. A new method of integrated digital volume correlation adapted to the spherical indentation test is presented to address these issues. It is proposed to couple in-situ experimental tests, DVC and numerical simulations. The key is to design a reduced kinematic basis, i.e. using very few degrees of freedom, yet being faithful to the kinematics of the test. The advantage of such an approach is that it may benefit from prior knowledge on the expected displacement field.

\section{Spherical indentation test}

Specimens used in the experiment are prepared from industrial plasterboard plates. The samples of the present study are cylinders $17 \mathrm{~mm}$ in diameter and $10 \mathrm{~mm}$ in height. For the in-situ experiment a spherical indenter (6 $\mathrm{mm}$ in diameter) is chosen. The indentation test is considered as quasi-static since a very low velocity is selected for the indenter (i.e. $5 \mu \mathrm{m} / \mathrm{s}$ ). The applied load and displacement are recorded and monitored during the test. The experimental setup is shown in Figure 1. The specimen is imaged at the initial state (i.e. without any applied load) and at nine levels of compressive load. After each step, the crosshead displacement is interrupted and a dwell time of $20 \mathrm{~min}$ is chosen to avoid blurring caused by displacement of the sample during the relaxation process. The experiment has been conducted on a Phoenix $\mathrm{V}$ tome $\mathrm{x}$ tomograph (90 KV-voltage, $200 \mu \mathrm{A}$ intensity, voxel size of $12 \mu \mathrm{m}, 1,920 \times 1,536$ pixel flat panel). 1,000 radiographs are acquired over a $360^{\circ}$ rotation for each scan. The total scan time lasts approximately 60 minutes.

Figure 2 shows the load-displacement curve of the test analyzed herein. A quasi-linear dependence of the applied load with the indentation depth is observed. The oscillations in the load-displacement response are due to repeated cycles of yield, collapse and densification. As the deformation progresses, the indenter is supported by a continuously increasing crushed zone of compacted plaster. A comparable load-penetration relationship was obtained for porous rocks [30].

Figure 3 shows 3D renderings of the reconstructed volumes of the sample in its reference configuration and at the eighth loading step. The compacted zone, where the microstructure is crushed due to the compression load, is clearly visible on the top part of the $3 \mathrm{D}$ rendering. The densified zone is located directly below the head of the indenter and a semi-ellipsoidal shape develops. However outside of the compacted region, the microstructure remains unaltered. As the load level increases, a larger crushed zone is formed under the indenter. After a certain load level, this crushed and compacted region acts as a virtually incompressible material that transfers the stresses to the surrounding intact plaster. Once the compaction zone is fully developed, the shape of the indenter is no longer relevant to the indentation process, which is totally controlled by the size and shape of the compaction zone. A similar phenomenon with 
a comparable shape was previously observed for highly porous refractory bricks (i.e. $65 \%$ porosity [29]), artificial porous rock [30] and aluminium foam [32, 31].

The aim of the next section is to introduce the global DVC approach to be used to measure displacement and strain fields in this type of experiment. One of the challenges is related to the fact that very small strain levels occur outside the crushed zone, and the latter is not directly accessible to standard DVC.

\section{Global DVC}

In the present study, a global approach to DVC [19] is used. The displacement field is decomposed over finite element shape functions. It allows for a direct coupling with numerical simulations [21, 22]. If the reference (respectively, deformed) image is represented by a three-dimensional gray level-valued field, $f(\underline{x})$, (respectively, $g(\underline{x})$ ), the gray level conservation requires that

$$
f(\underline{x})=g(\underline{x}+\underline{u}(\underline{x}))
$$

where $\underline{u}$ denotes the unknown displacement field. Measuring a displacement field consists of minimizing the square of the correlation residual $\phi_{c}$

$$
\phi_{c}=|g(\underline{x}+\underline{u}(\underline{x}))-f(\underline{x})|
$$

The minimization of $\phi_{c}^{2}$ is a nonlinear and ill-posed problem. In particular, if no additional information is available, it is impossible to determine the displacement for each voxel independently since there are three unknowns for a given (scalar) gray level. For these reasons, a weak formulation is preferred. After integration over the whole Region Of Interest (ROI) $\Omega$, the global correlation residual $\Phi_{c}^{2}$ reads

$$
\Phi_{c}^{2}=\frac{1}{2} \int_{\Omega}(g(\underline{x}+\underline{u}(\underline{x}))-f(\underline{x}))^{2} \mathrm{~d} \underline{x}
$$

in which the displacement field is expressed in a (chosen) basis as

$$
\underline{u}(\underline{x})=\sum_{i} u_{n} \underline{\psi}_{n}(\underline{x})
$$

where $\underline{\psi}_{n}$ are (chosen) vector fields, and $u_{n}$ the associated degrees of freedom. The measurement problem then consists of minimizing $\Phi_{c}^{2}$ with respect to the unknowns $u_{n}$. A modified Newton iterative procedure is followed to circumvent the nonlinear character of the minimization problem. Let $\underline{u}^{i}$ denote the displacement at iteration $i$, and $\{\boldsymbol{u}\}^{i}$ the vector containing all the unknown degrees of freedom. By assuming small increments $\mathrm{d} \underline{u}=\underline{u}^{i+1}-\underline{u}^{i}$ of the solution, a Taylor expansion is used to linearize $g\left(\underline{x}+\underline{u}^{i+1}(\underline{x})\right) \approx g\left(\underline{x}+\underline{u}^{i}(\underline{x})\right)+\mathrm{d} \underline{u}(\underline{x}) \cdot \underline{\nabla} f(\underline{x})$ and $\partial \Phi_{c}^{2} / \partial\{\underline{u}\}^{i}$ is recast in a matrix-vector product as

$$
\frac{\partial \Phi_{c}^{2}}{\partial\{\boldsymbol{u}\}^{i}}=[\boldsymbol{M}]\{\mathrm{d} \boldsymbol{u}\}-\{\boldsymbol{b}\}^{i}=\{\mathbf{0}\}
$$

with

$$
M_{m n}=\sum_{\Omega}\left(\underline{\nabla} f(\underline{x}) \cdot \underline{\psi}_{m}\right)\left(\underline{\nabla} f(\underline{x}) \cdot \underline{\psi}_{n}\right)
$$

and

$$
b_{m}^{i}=\sum_{\Omega}\left(\underline{\nabla} f(\underline{x}) \cdot \underline{\psi}_{m}\right)\left(f(\underline{x})-g\left(\underline{x}+\underline{u}^{i}(\underline{x})\right)\right)
$$


In the above equation the corrected deformed image, $g\left(\underline{x}+\underline{u}^{i}(\underline{x})\right)$ is computed with a trilinear gray level interpolation.

At this level of generality, many choices can be made to measure displacement fields. When dealing with volumes, eight-noded elements with classical finite element bases can be used [19]. The latter is referred to as C8-DVC (i.e. global DVC with 8-noded cubes). In the sequel, C8-DVC will be used since no displacement discontinuity is sought. Consequently, the region of interest is discretized with finite elements for which the displacement field is interpolated with trilinear shape functions. Global C8-DVC is first used to measure 3D displacement fields in the bulk of the sample. A regular mesh with $\mathrm{C} 8$ elements is used. The displacement field at the $n$-th load level is assessed by correlating the $n$-th volume with the initial volume (unloaded state considered as the reference). The region of interest has a size of $576 \times 576 \times 256$ voxels (or $6.9 \times 6.9 \times 3.07 \mathrm{~mm}$ ). The size of the $\mathrm{C} 8$ elements was chosen to be 8 voxels (i.e. $96 \mu \mathrm{m}$, which is of the order of the wall size). This choice resulted in a little more than 360,000 unknown kinematic degrees of freedom.

The measured displacement field is shown in Figure 4. As the crushed region has lost its microstructure, this region is masked from the ROI and the displacement field remains undetermined there. A simple interpolation is used to compute the residual field shown in Figure 4(a). In the latter, normalized residuals are shown, namely $\phi_{c} / \Delta f$, where $\Delta f$ denotes the dynamic range of the ROI in the reference configuration. It was chosen here to compute a harmonic interpolation, i.e. solving $\underline{\nabla}^{2} \underline{u}=0$ inside the mask, and matching the computed values on the boundary (Dirichlet-type boundary conditions). Let us stress that this interpolation is not used for any measurement, but just to reveal the crushed region. From the latter 3D image the region where plaster has been crushed is easily distinguished as its boundary is sharp. The mask is adjusted as being the smallest semi-ellipsoid that contains the crushed region (Figure 4(a)). Because of the uncertainty level of the reconstructed images themselves and the modest range of observed displacement magnitude outside the crushed zone, the measurement appears as too noisy to allow for the determination of strains. Displacement fluctuations are clearly visible since the dynamic range is very small (i.e. less than 2 voxels) as outside the compacted volume plaster remains mostly elastic. Many of these fluctuations are due to measurement uncertainties associated with very small element sizes.

The uncertainty on the measurement of the displacement fields is estimated in a region very remote from the compacted area where strains are very small. After removal of the rigid body motions, the root mean square fluctuations of the displacement field is of the order of 0.4 voxel or $\approx 5 \mu \mathrm{m}$. This very high level is due to the high volume fraction of pores $(\approx 75 \%)$ in combination with small element sizes. As a consequence, the strain uncertainty for such a small element size is quite large $\left(\right.$ i.e. $5 \times 10^{-2}$ ). This first analysis shows that even with very small element sizes, global C8-DVC provides a good first displacement estimate. However, it is corrupted by measurement uncertainties and strain evaluations cannot be achieved faithfully at such a small scale.

To go beyond the previous results, the crucial point is to select the appropriate discretization of the sought displacement field. Large elements possibly allow for a low uncertainty in the measured displacement at the expense of a poor spatial resolution. Conversely, fine meshes induce better spatial resolutions, but because of the fewer voxels considered per element, displacement uncertainties are larger. In addition, reconstruction artifacts are inevitably present in the image [35] and thus very small element sizes can hardly be used. Hence, the analysis of the displacements results from a compromise between spatial resolution and displacement uncertainty [36, 37]. Both quantities are influenced by the image texture and the "complexity" of the displacement field [38].

Complexity is to be understood here as the ability to capture the actual displacement field with a specific discretization. Because in the elastic domain, strains are expected to decay as 
$1 / r^{2}$ where $r$ is the distance to the indentor center, a significant discretization error is expected for coarse meshes in particular close to the indentor or the boundary of the crushed zone. This very rapid decrease of strain with $r$ constitutes an intrinsic inhomogeneity (not due to the random microstructure) that is challenging for DVC. Further, Gaussian filtering (especially at boundaries) although commonly used in Digital Image Correlation cannot be trusted in the studied case, as even in the absence of noise, it will severely affect the strain field. What would be needed is a filter that preserves a mechanically meaningful component and only damps out spurious fluctuations. Recently, Leclerc et al. [37] proposed such a filter in so-called regularized DIC or DVC, where rather than using a Gaussian filter, a mechanical modeling can be used to assist DVC. This procedure could have been used here but the following procedure proposes an alternative route to circumvent the difficulty of using a fine mesh (and hence a large uncertainty) in spite of the fact that the displacement fields in indentation tests are expected to display steep gradients.

\section{Reduced basis in DVC}

The idea is to design a reduced basis, which is adapted to the indentation test and thus allows for an accurate description of the test kinematics especially at the interface of the crushed zone and the remainder of the sample that is assumed to behave elastically. The fact that some $a$ priori information on the sought displacement fields is available allows for a significant reduction in the number of degrees of freedom. Because the studied domain excludes the crushed zone, and that no sign of deviation from linear elasticity is observed, it is assumed that the behavior remains linearly elastic in the entire domain studied by DVC. The porous gypsum is treated as a homogeneous and isotropic solid, with Poisson's ratio $\nu=0.15$ (note that the Young's modulus is not needed). In the present case, the displacement fields have a low sensitivity to Poisson's ratio when it is varied between 0 and 0.2 .

Moreover, the boundary conditions are known over a large part of the domain boundary, namely, the face opposite to the indentation undergoes essentially rigid body motions that can be measured, all other faces are free, and finally only the displacements or forces are unknown on the surface, $\mathcal{S}$, of the masked crushed region. Would the latter be known that the computation of the displacement field in the entire domain would be obtained from a 3D linear elastic simulation. (It is observed that the crushed zone underneath the indentor constitutes an additional difficulty as compared to a rigid indentor where the displacement field over $\mathcal{S}$ would be a mere translation.) The spirit of the proposed approach is to design a parameterization of the displacement field on $\mathcal{S}$ based on few degrees of freedom. Each of these unknown parameters $i$ corresponds to a $3 \mathrm{D}$ displacement field $\underline{\psi}_{i}(\underline{x})$ computed from a commercial FE software (Abaqus ${ }^{\circledR}$ in the present case). This set of fields $\underline{\psi}_{i}(\underline{x})$ is the basis of kinematic fields used in DVC, as explicitly written in Equation (4). The measured amplitudes, $a_{i}$, are also used to compute the actual displacement at the boundary of the masked region. It is worth noting that the computation time of C8-DVC is much higher than that of the reduced basis because of the number of unknowns has been drastically reduced (from 360,000 to 9 in the present case).

The boundary conditions used hereafter consist of (i) a rigid body motion on the face opposite the indentation (adjusted from a coarse C8-DVC analysis), (ii) traction-free surfaces over all other surfaces apart from the boundary of the crushed domain, $\mathcal{S}$ and (iii) on the $\mathcal{S}$ boundary, the displacement is sought as a kind of spherical harmonics expansion (truncated at low orders) for each component. More precisely, the library of fields used to describe the 
displacement is first restricted to axisymmetric modes written as

$$
\left\{\begin{array}{l}
u_{x}\left(a_{i}, \varphi, \theta\right)=v \cos (\varphi) \sin (\theta)+w \cos (\varphi) \cos (\theta) \\
u_{y}\left(a_{i}, \varphi, \theta\right)=v \sin (\varphi) \sin (\theta)+w \sin (\varphi) \cos (\theta) \\
u_{z}\left(a_{i}, \varphi, \theta\right)=v \cos (\theta)-w \sin (\theta)
\end{array}\right.
$$

where $\theta$ and $\varphi$ are respectively the polar and azimuthal angles. As shown in Figure $5, w$ is a the displacement along the radial $\underline{e}_{r}$ direction, and $v$ along $\underline{e}_{\theta}$. Both fields, $v$ and $w$ read

$$
\left\{\begin{array}{l}
v\left(\theta ; a_{i}\right)=a_{1}+a_{2} \cos (\theta)+a_{3} \cos (2 \theta)+a_{4} \cos (3 \theta) \\
w\left(\theta ; a_{i}\right)=a_{5} \sin (\theta)+a_{6} \sin (2 \theta)+a_{7} \sin (3 \theta)
\end{array}\right.
$$

where $a_{i}(i=1, \ldots, 7)$ denote the amplitudes of the fields to be identified. It revealed necessary to enrich this basis by two additional fields to account for a breakdown of axisymmetry that was not anticipated initially. It was chosen to impose over $\mathcal{S}$ a uniform displacement along $x$ and $y$, respectively for modes 8 and 9 . These two fields account for a lateral motion of the indentor in addition to its normal penetration. Let us also note that the rigid body motion measured on the face opposite the indentor, as shown in Figure 6(a), is not axisymmetric either. This spurious motion may be due to a compliant indentor axis combined with slight heterogeneities in the to-be-crushed plaster. Let us emphasize the fact that beyond the specific choice made herein, the proposed approach is extremely flexible and can easily be enriched to account for whatever effect could be detected and modeled, such as the present breakdown of symmetry, or possibly the occurrence of an indentation crack (not observed hereafter). Last, mixing Dirichlet and Neumann boundary conditions for different fields $\psi_{i}$ is totally transparent in the present setting.

An overview of the mesh of the FE model used to compute the displacement basis, which uses tetrahedral elements, is shown in Figure 6. Close to the boundary of the crushed zone, the element size is of the order of $100 \mu \mathrm{m}$ (or 8 voxels). In the same figure, two examples of displacement fields generated by Abaqus ${ }^{\circledR}$ are presented.

From the finite element simulations, nine displacement fields defined at the location of each voxel from the finite element shape function are obtained (so that no mesh is needed anymore for the subsequent DVC analyses). However, as the displacement of each field spreads over the entire domain, the problem to be solved at each time step is global and involves a full correlation matrix $[\boldsymbol{M}]$. Its size being very modest $($ i.e. $9 \times 9)$ the correlation calculation is not the limiting step. Convergence is observed to be excellent, and the level of mean correlation residuals $(5.14 \%)$ is comparable to that observed with the initial C8-DVC basis (5.06\%). This result validates the chosen kinematic basis. Let us emphasize the fact that the number of kinematic degrees of freedom has been reduced from about 360,000 to 9. Moreover for any combination of amplitudes $\left\{a_{i}\right\}$, the resulting displacement field obeys the equilibrium and constitutive equations as well as the traction-free or prescribed rigid motion at the basis of the specimen.

The displacement fields measured with the reduced kinematic basis are shown in Figure 7 . Comparing the displacement fields obtained by the two approaches, it is noted that the same range of displacement is measured. However most of the fluctuations are filtered out, yet the long wave features are still captured. The uncertainty on the measurement of the displacements fields is estimated in the same remote region from the compacted area. The standard displacement uncertainty is of the order of 0.03 voxel or $\approx 0.3 \mu \mathrm{m}$. Comparing the latter with results obtained by $\mathrm{C} 8$-DVC $(\approx 5 \mu \mathrm{m})$, there is a marked decrease in the standard displacement uncertainty. Similarly, the strain uncertainty is less than $2 \times 10^{-3}$, i.e. at least one order of magnitude lower than previously observed.

Figure 8 shows the deviatoric and volumetric strain fields in the same plane as they are estimated with both procedures, namely, reduced basis (a) and C8 approach (b). It is worth 
noting that the dynamic range has to be increased for the standard C8 approach when compared with the results obtained with the reduced basis approach. For C8-DVC, the fluctuations are mainly associated with measurement uncertainties as opposed to the new approach that is able to capture the strain gradients. Note also that the deviatoric strain is a positive quantity and hence its average value for a noisy displacement field will remain well above the average of a noiseless field.

From the latter determination, the elastic strain or stress components, in particular on the boundary of the crushed domain, can be computed more reliably. Figure 9 shows the results obtained with the optimal amplitudes. The displacement field is presented in Figure 9(a). It is observed that the optimal displacement field is not axisymmetric. Figure $9(\mathrm{~b})$ shows the maximum principal strain fields. The proposed procedure reveals a much more complex displacement field than what is traditionally assumed for perfect spherical indentation. The results display very large differences between the identified strain (or stress) distribution and the expected theoretical distribution. In addition, this new strategy allows very small strains to be measured.

The choice for the reduced basis may appear as somewhat arbitrary. It has to be simple (to limit uncertainty) and yet representative (to be able to account precisely for the actual displacement field). Representativeness is checked by considering the residual field, ideally comparable to that achieved with the first C8 procedure. Simplicity means that a minimum number of fields is to be used. A natural basis is provided by a multipolar expansion, akin to spherical harmonics. This expansion has been further simplified to account for axisymmetry, and truncated to low orders. As discussed above, the first choices were not representative (i.e. led to large correlation residuals) until deviation from axisymmetry was introduced. This choice is certainly not unique, and it is possible that other designs would lead to smaller numbers of fields. What is important is that the above choice does fulfill the above two criteria as shown below.

In order to quantify the sensitivity of the results to the choice of displacement fields of the reduced basis, two other choices are considered. The measured rigid body motion is still prescribed on the bottom of the sample and different combinations of numerical fields (see Equation (9)) are prescribed on the boundary of the crushed zone. For the basis that contains at least the first field of the series $\left(a_{1}\right)$ and the two shear fields (i.e. of amplitudes $a_{8}$ and $a_{9}$ ), the results are very close to those obtained with 9 fields. The mean correlation residual is equal to $5.16 \%$, level very close to the values found by C8-DVC, $5.06 \%$, and with the first set of amplitudes (i.e. $a_{1}$ to $a_{9}$ ) $5.14 \%$.

Any attempt to reduce further the number of fields (less than three fields, i.e. of amplitudes $a_{1}, a_{8}$ and $a_{9}$ ) results in a significant degradation of the residual level, showing that the proposed restriction is inappropriate. Let us emphasize on the fact that the strong assumptions that are made to reduce the kinematic basis are not to remain unchallenged. The residual field and mean value provide objective quality estimates with appropriate spatial resolution to judge whether the initial assumptions can be deemed acceptable, or if they are to be revisited or relaxed.

\section{Conclusions and perspectives}

In situ mechanical tests within a tomograph, and in particular indentation tests, are seen to be well suited for an exhaustive characterization of the full 3D kinematics, and hence identification from such DVC results is appealing. However, material microstructure, tomography artifacts, test inhomogeneity may all lead to large uncertainties. The proposed methodology is a way to alleviate those difficulties through the exploitation of known prior information within digital volume correlation. 
The tomographic observations show that indentation proceeds by the growth of the crushed domain underneath the indentor, while the remainder of the medium can be considered as elastic. This a priori information is used to design a new DVC approach based on a reduced basis exploiting the knowledge of the elastic behavior and the boundary conditions. Each field of the basis is the result of a finite element simulation outside the crushed zone. The few remaining unknowns are determined by image registration.

A very significant uncertainty reduction is observed although the mean displacements remain of the same order of magnitude. It is worth noting that with the proposed strategy the strain and stress fields are accessible even if their level is small. This is possible because the measured displacement fields are not only kinematically admissible but also statically admissible.

This novel DVC strategy opens new pathways for the identification of mechanical properties. This type of approach is very generic and can be extended to other experimental configurations and material behaviors. It uses the finite element code as a way of generating kinematic bases. The amplitudes associated with each considered field are then evaluated via a global approach to digital volume correlation. This approach is non intrusive as any finite element code can be used as is.

The next step of the present analysis will consist of the identification of the behavior in the vicinity of the boundary between the crushed plaster and the pristine material. Full benefit will be made by the fact the stress and strain fluctuations close to the interface are measured very accurately with very fine discretizations.

\section{Acknowledgements}

The authors wish to thank Saint-Gobain Recherche for supporting this research project, and particularly Xavier Brajer and René Gy. AB is also financially supported by ANRT through contract no. $2010 / 567$.

\section{References}

[1] Vimmrová, A., Keppert, M., Svoboda, L., Černý, R. (2011) Lightweight gypsum composites: Design strategies for multi-functionality. Cem. \& Concr. Comp., 41, 557-546

[2] Murat, M., Pusztaszeri, L., Gremion, M. (1974) Corrélation texture cristalline-propriétés mécaniques de plâtres durcis, étude préliminaire. Mat. $\&$ Constr., 47, 337-385

[3] Coquard, P. (1992) Résistance mécanique des plâtres secs et humides. PhD thesis, AixMarseille University (France)

[4] Meille, S. (2001) Etude de comportement mécanique du plâtre pris en relation avec sa microstructure. PhD thesis, INSA de Lyon (France)

[5] Sakji, M.S.(2006) Modélisation probabiliste et validation expérimentale du transfert thermique et du comportement thermomécanique avec endommagement d'une plaque multicouche carton-plâtre-carton soumise au feu. PhD thesis, Paris Est Marne-La-Vallée University (France)

[6] Benouis, A. (1995) Comportement mécanique des ouvrages en plaques de plâtre sur ossature métallique. PhD thesis, Ecole Nationale des Ponts et Chaussées (France)

[7] Baruchel, J., Buffière, J.-Y., Maire, E., Merle, P., Peix, G. (2000) X-Ray Tomography in Material Sciences. Hermes Science, Paris (France) 
[8] Bay, B.K., Smith, T.S., Fyhrie, D.P., Saad, M. (1999) Digital Volume Correlation: Threedimensional Strain Mapping Using X-ray Tomography. Exp. Mech., 39, 217-26

[9] Maire, E., Caty, O., King, A., Adrien, J. (2009) X Ray Tomography Study of Cellular Materials: Expriments and Modelling. Mechanical Properties of Cellular Materials IUTAM Bookseries, Springer, 12, 35-42

[10] Buffière, J.Y., Maire, E., Clotens, P., Lormand, G., Fougères, R. (1999) Characterization of internal damage in a MMCp using X-ray synchrotron phase contrast mincrotomography. Acta Mat., 47, 1613-25

[11] Ferrié, E., Buffière, J.-Y., Ludwig, W., Gravouil, A., Edwards, L. (2006) Fatigue crack propagation: in situ visualization using X-ray microtomography and 3D simulation using the extended finite element method. Acta Mat., 5, 1111-1122

[12] Guvenilir, A., Breunig, T.M., Kinney J.H., Stock, S.R. (1997) Direct observation of crack opening as a function of applied load in the interior of a notched tensile sample of Al-Li2090. Acta Mat., 45, 1977-1987

[13] Elliot, J.A., Windele, A.H., Hobdell, J.R., Eeckhaut, G., Olman, R.J., Ludwig, W., Boller, E., Cloetens, P., Baruchel, J. (2002) In-situ deformation of an open-cell flexible polyurethane foam characterized by 3D computed mincrotomography. J. Mat. Sci., 37, $1547-1555$

[14] Buffière, J.-Y., Maire, E., Adrien, J., Masse, J.P and Boller, E. (2010) In Situ Experiments with X ray Tomography: An Attractive Tool for Experimental Mechanics. Exp. Mech., 50, 289-305

[15] Youssef, S., Maire, E., Gaertner, R. (2005) Finite element modelling of the actual structure of cellular materials determined by X-ray tomography. Acta Mat., 53, 719-730

[16] Smith, T.S., Bay, B.K., Rashid, M. (2002) Digital volume correlation including rotational degrees of freedom during minimization. Exp. Mech., 42, 272-278

[17] Lenoir, N., Bornert, M., Desrues, J., Bésuelle, P., Viggiani, G. (2007) Volumetric digital image correlation applied to X-ray microtomography images from triaxial compression tests on argillaceous rock. Strain, 43, 193-205

[18] Sutton, M.A., Wolters, W.J., Peters, W.H., Ranson, W.F. and McMeill, S.R. (1983) Determination of displacements using an improved digital correlation method. Image Vis. Comput., 1, 133-139

[19] Roux, S., Hild, F., Viot, P., Bernard, D. (2008) Three-dimensional image correlation from X-ray computed tomography of solid foam. Comp. Part A, 39, 1253-1265

[20] Hild, F., Maire, E., Roux, S., Witz, J.-F. (2009) Three dimensional analysis of a compression test on stone wool. Acta Mat., 7, 3310-3320

[21] Limodin, N., Réthoré, J., Buffière, J.-Y., Gravouil, A., Hild, F., Roux, S.(2009) Crack closure and stress intensity factor measurements in nodular graphite cast iron using 3D correlation of laboratory X ray microtomography images. Acta Mat., 57, 4090-4101

[22] Rannou, J., Limodin, N., Réthoré, J., Gravouil, A., Ludwig, W., Baïetto-Dubourg M.C., Buffière, J.-Y., Combescure, A., Hild, F., Roux, S. (2010) Three dimensional experimental and numerical multiscale analysis of a fatigue crack.Comput. Meth. Appl. Mech. Eng., 199, $1307-1325$

[23] Chatelain, E. (2011) Comportement mécanique de mousses fragiles. Projet de fin d'études. INSA de Lyon (France)

[24] Shaw, M.C, Sata, T. (1966) The plastic behavior of cellular materials. Int. J. Mech. Sci., $8,469-472$ 
[25] Wilsea, M., Johnson, K.L, Ashby, M.F. (1975) Indentation of foamed plastics. Int. J. Mech. Sci, 17, 457-460

[26] Lawn, B.R. (1998) Indentation of Ceramics with Spheres: A Century after Hertz. J. Am. Ceram. Soc, 81 [8] 1977-94

[27] Kumar, S.P., Ramachandra, S., Ramamurty, U. (2003) Effect of displacement-rate on the indentation behavior of an aluminum foam. Mat. Sci. Eng, A347, 330-337

[28] Onck, P.R., Andrews, E.W., Gibson, L.J. (2011) Size effects in foams: Experiments and modeling. Pro. Mat. Sci, 56, 109-138

[29] Suárez-Rivera, F.R., Cook, N.G., Cooper, G.A., Zheng, Z. (1990) Indentation by pore collapse in porous rocks. Proc. 31st US Symp. on Rock Mech., 671-678

[30] Leite, M.H, Ferland, F. (2001) Determination of unconfined compressive strength and Young's modulus of porous materials by indentation tests. Eng. Geolog., 59, 267-280

[31] Kádár, Cs., Maire, E., Borbély, A., Peix, G., Lendvai, J., Rajkovits, Zs. (2004) X-ray tomography and finite element simulation of the indentation behavior of metal foams. Mat. Sci. Eng, A387-389, 321-325

[32] Smith, T.S., Bay, B.K. (2001) Experimental Measurement of Stains using Digital Volume Correlation. Nontraditional Methods of Sensing Stress, Strain, and Damage in Materials and Structures: Second Volume, ASTM STP 1323, G.F., Lucas, P.C. McKeighan, and J.S. Ransom, Eds., American Society for Testing and Materials, West Conshohocken, Pa (USA)

[33] Bay, B.K. (2008) Methods and applications of digital volume correlation. J. Strain Analysis, 43, 745-760

[34] Mostafavi, M., McDonald, S.A., Mummery, P.M., Marrow, T.J. (2013) Observation and quantification of three-dimensional crack propagation in poly-granular graphite. Eng. Fra. Mech, 110, 410-420

[35] Davis, G.R, Elliot, J.C. (2006) Artefacts in X-ray microtomography of materials. Mat. Sci. Eng, 22, 1011-1018

[36] Bergonnier, S., Hild, F., Roux, S. (2005) Digital image correlation used for mechanical tests on crimped glass wool samples. J. Strain Analysis, 40(2), 185-197

[37] Leclerc, H., Périé, J.N., Hild, F., Roux, S. (2012) Digital Volume Correlation: What are the limits to the spatial resolution? Mech. ES Indus., 13, 361-371

[38] Réthoré, J., Hild, F., Roux, S. (2007) Shear-band capturing using a multiscale extended digital image correlation technique. Comp. Meth. Appl. Mech. Eng, 196, 5016-5030 


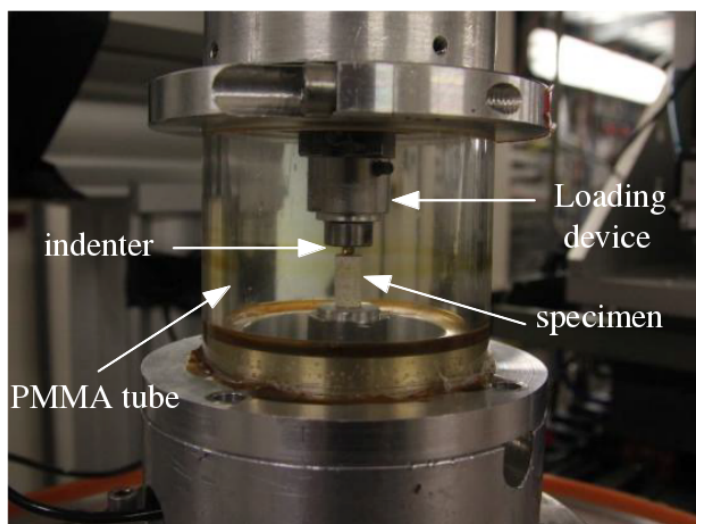

Figure 1: Specimen within the loading rig

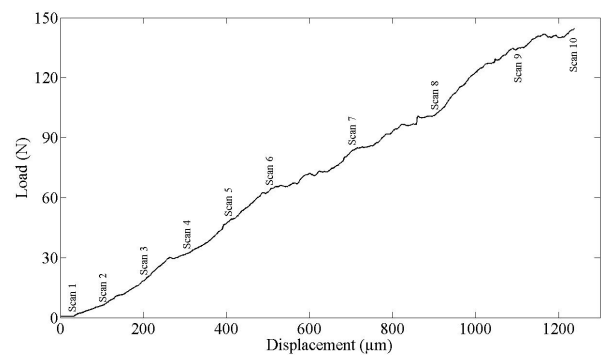

Figure 2: Load-displacement plot of a test with ten scans

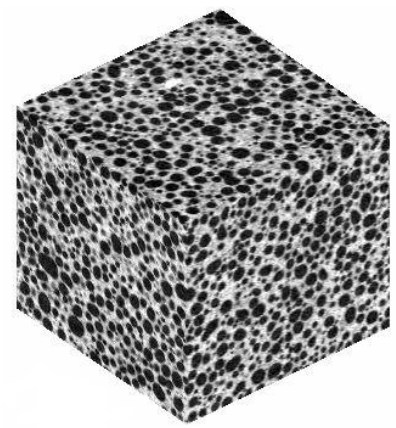

(a)

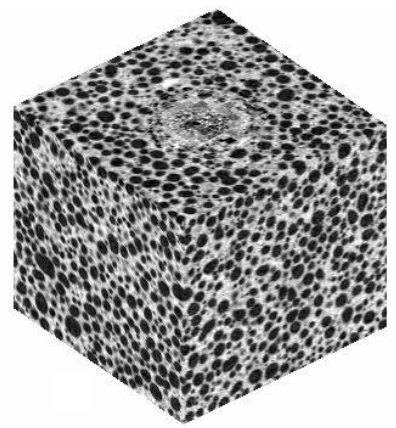

(b)

Figure 3: 3D renderings of the observed volume of interest. (a) Reference configuration. (b) Deformed configuration where the crushed region beneath the indenter (upper face) can be clearly observed 


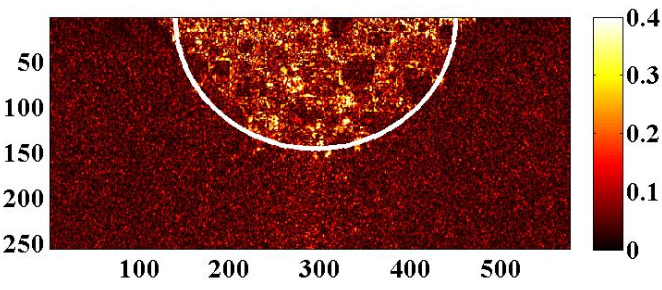

(a)

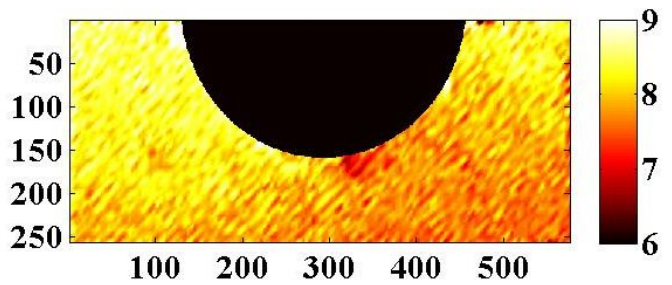

(c)

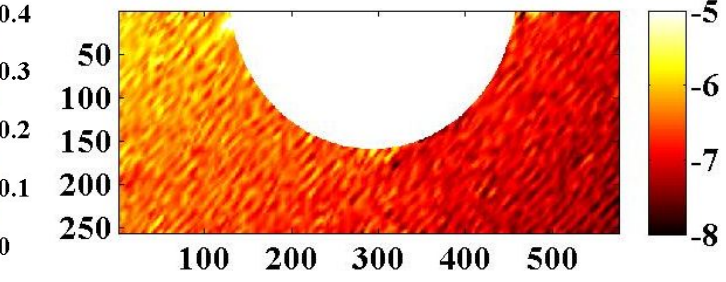

(b)

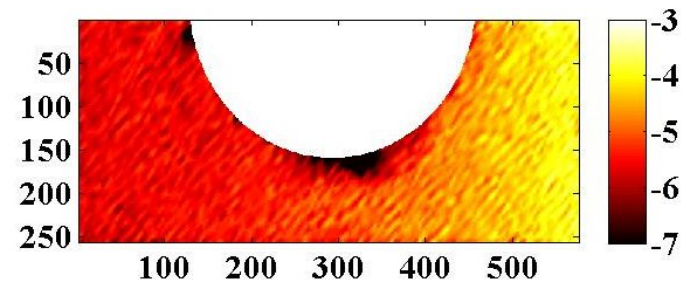

(d)

Figure 4: Correlation results with a C8-DVC approach for the eighth loading step. (a) Normalized correlation residual field $\phi_{c} / \Delta f$ in the $y-z$ plane at $x=288$ voxel. The white line depicts the boundary of the crushed zone. Corresponding $u_{x}$ (b), $u_{y}$ (c) and $u_{z}$ (d) displacement fields expressed in voxels $(1$ voxel $\leftrightarrow 12 \mu \mathrm{m})$

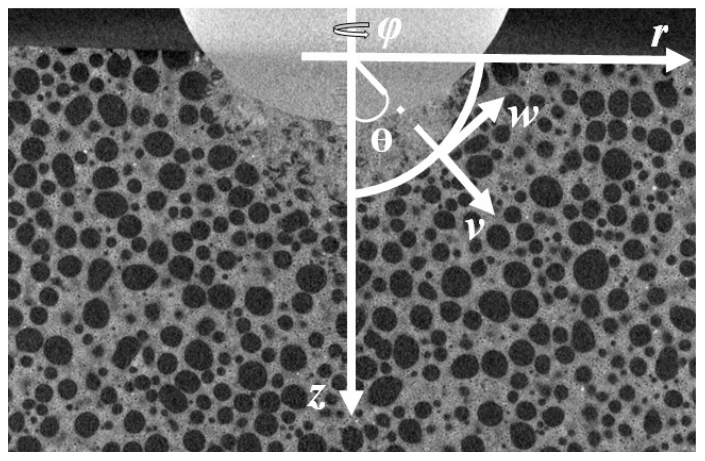

Figure 5: Spherical coordinates system 


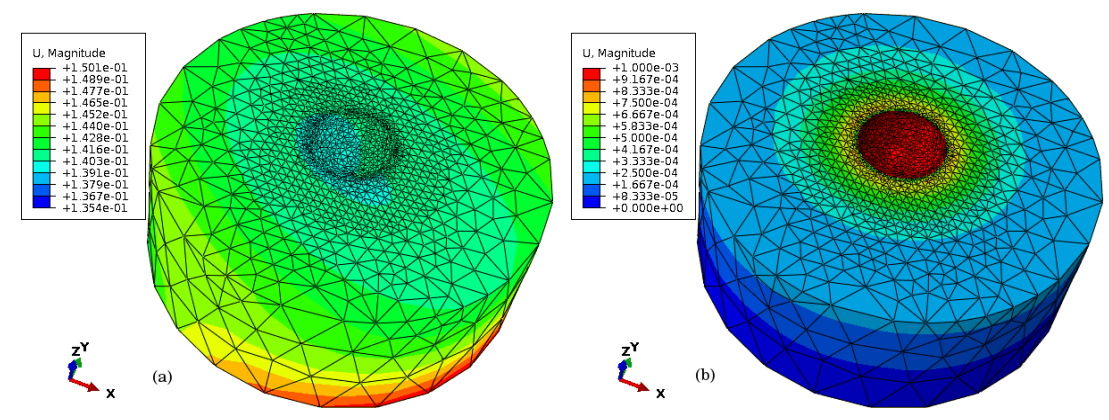

Figure 6: Examples of displacements fields generated by Abaqus ${ }^{\circledR}$ and colored by the magnitude of the displacement for the eighth loading step. (a) Rigid body motion field. (b) Displacement field corresponding to the first amplitude $a_{i}=\delta_{i j}$ with $j=1$. The displacement fields are expressed in $\mathrm{mm}$

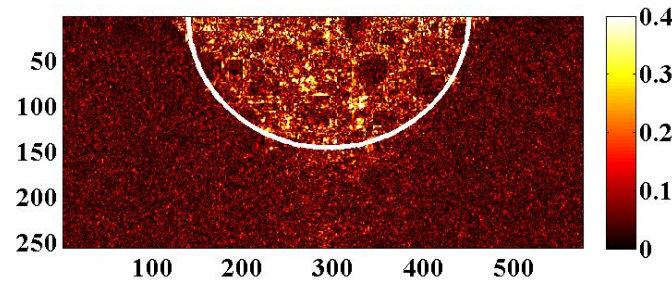

(a)

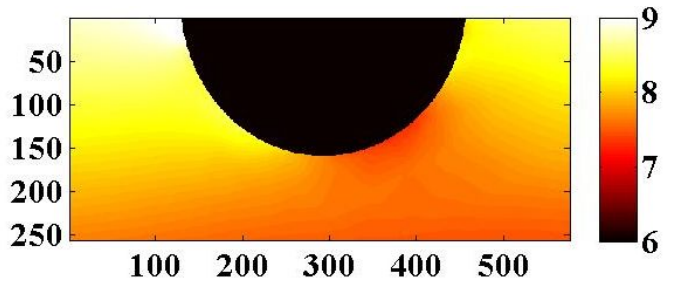

(c)

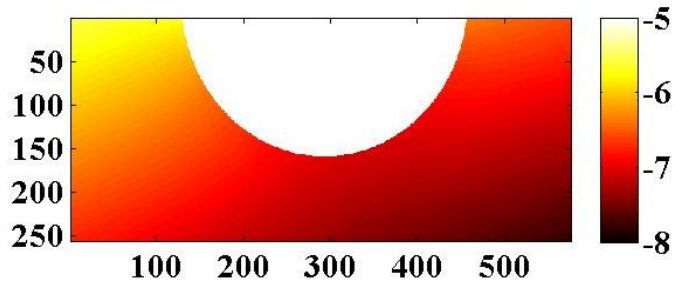

(b)

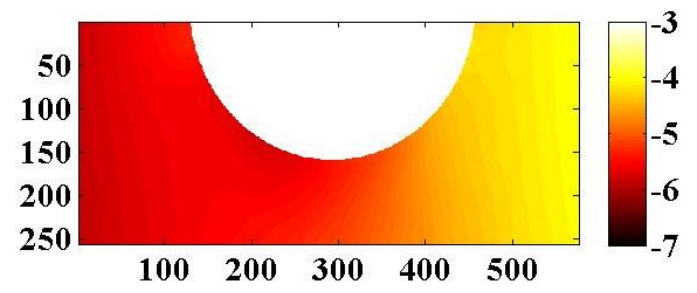

(d)

Figure 7: Displacements fields measured with a reduced basis for the eighth loading step. (a) Normalized correlation residual field $\phi_{c} / \Delta f$ in the $y-z$ plane at $x=288$ voxel. The white line depicts the boundary of the crushed zone. (b) Corresponding $u_{x}$ (b), $u_{y}$ (c) and $u_{z}$ (d) displacement fields when expressed in voxels ( 1 voxel $\leftrightarrow 12 \mu \mathrm{m}$ ) 


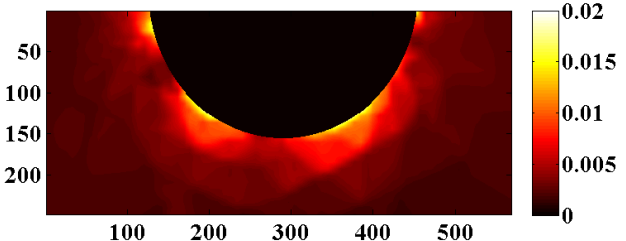

(a)

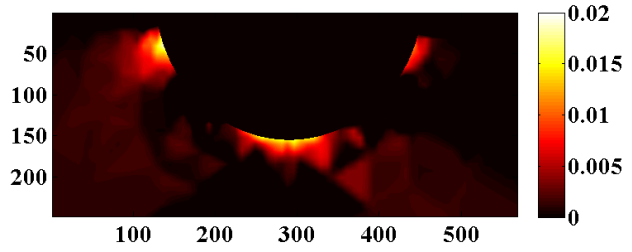

(c)

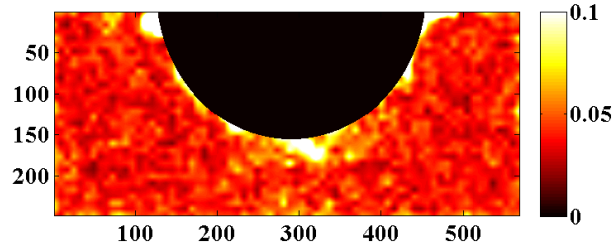

(b)

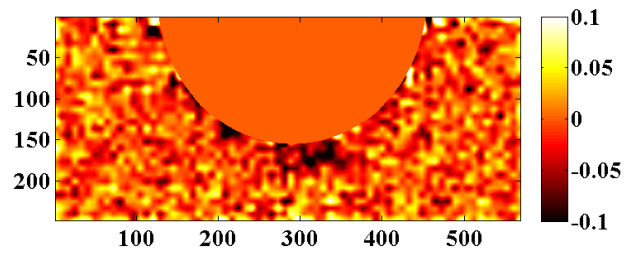

(d)

Figure 8: Strain maps for the eighth loading step. Deviatoric strain map shown in the $y-z$ plane for the reduced basis approach (a) and the corresponding $\mathrm{C} 8$ result (b). Volumetric strain map shown in the $y-z$ plane for the reduced basis approach (c) and the corresponding C8 result (d)
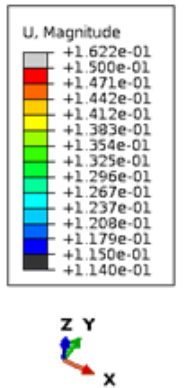

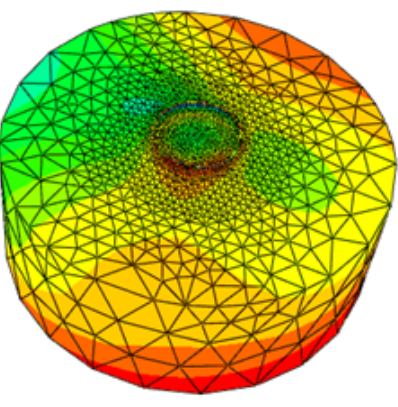

(a)

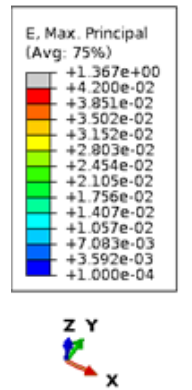

Figure 9: Correlation results with the optimal amplitudes for the eighth loading step. (a) Magnitude of displacement field. (b) Maximum principal strain field 